
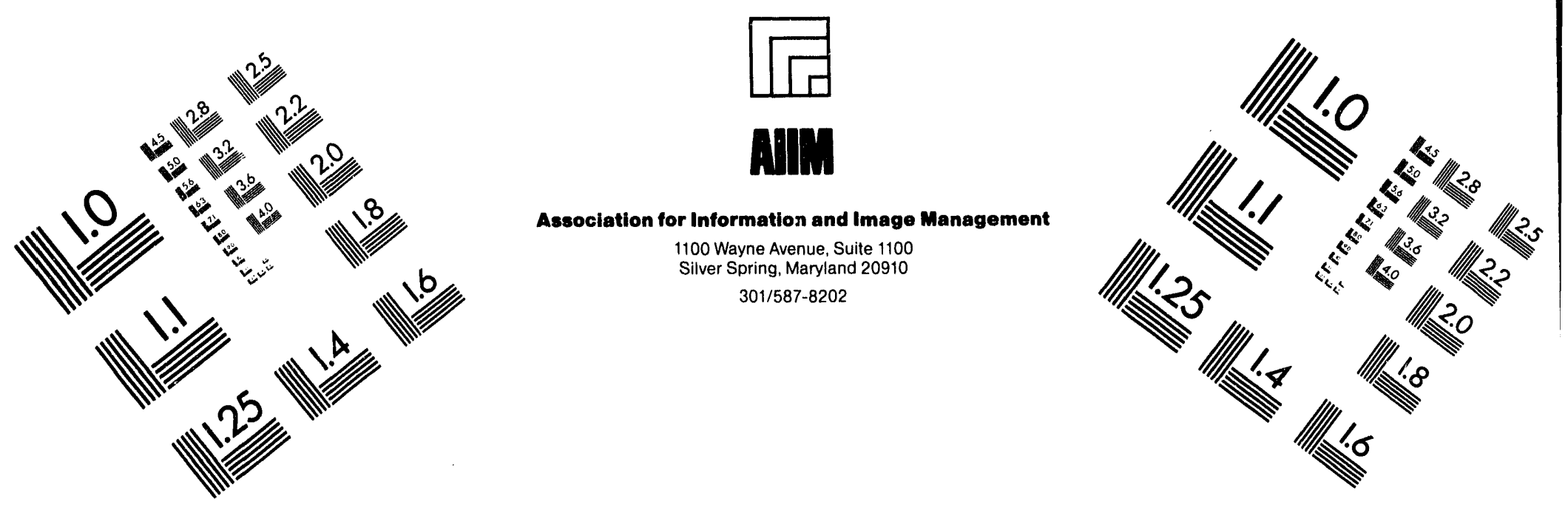

\title{
Centimeter
}

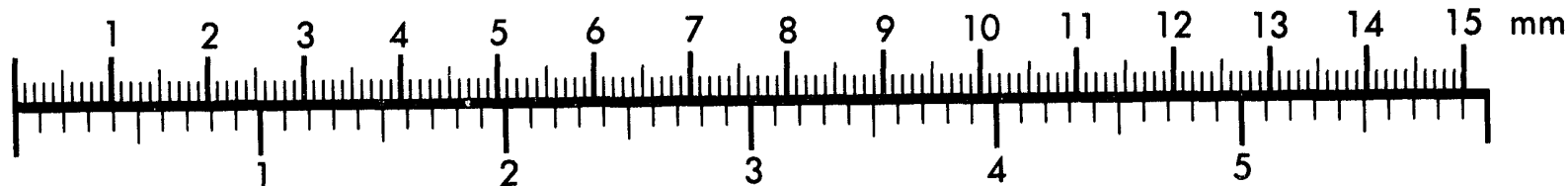
Inches
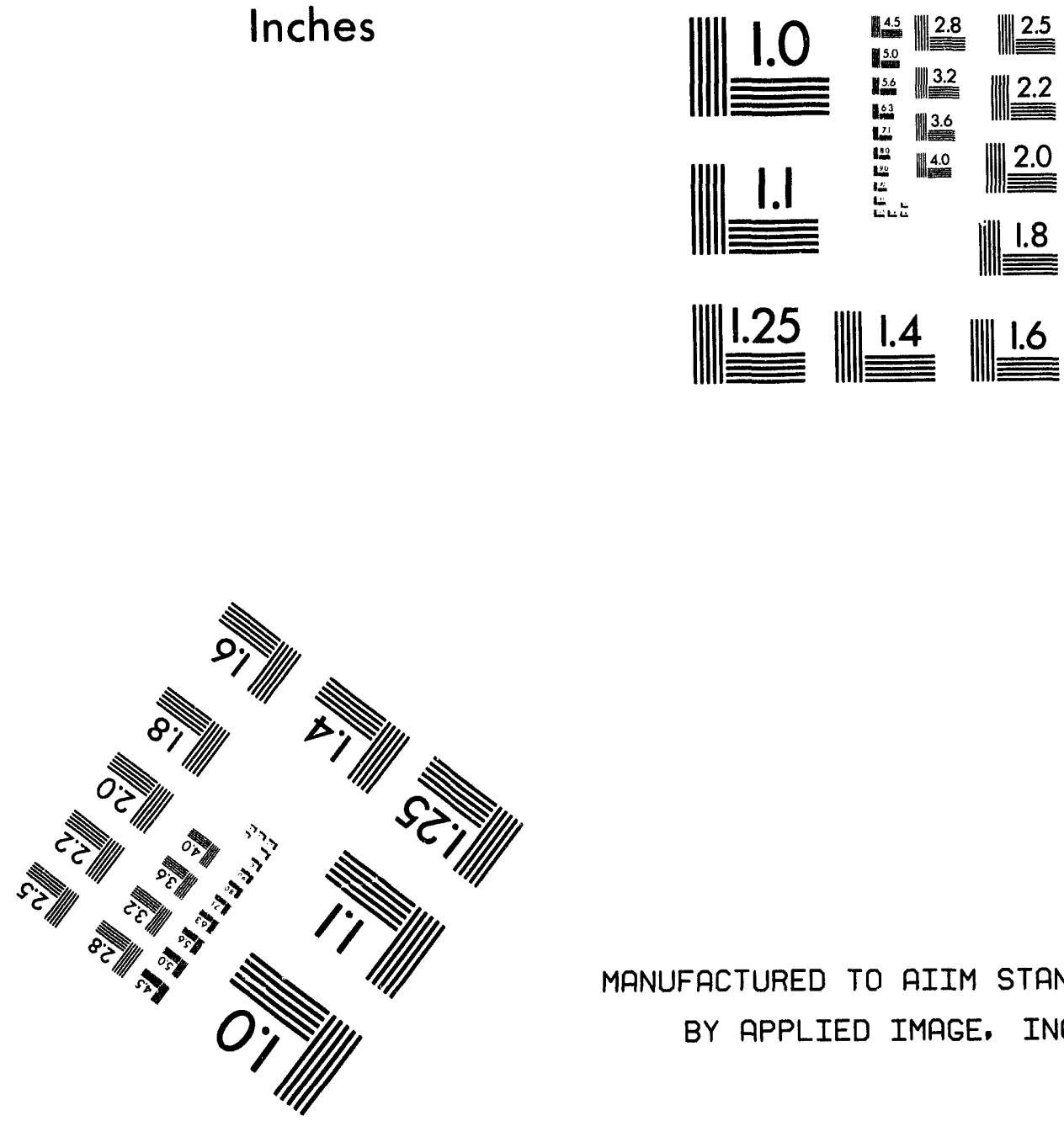

MANUFACTURED TO AIIM STANDARDS

BY APPLIED IMAGE, INC.

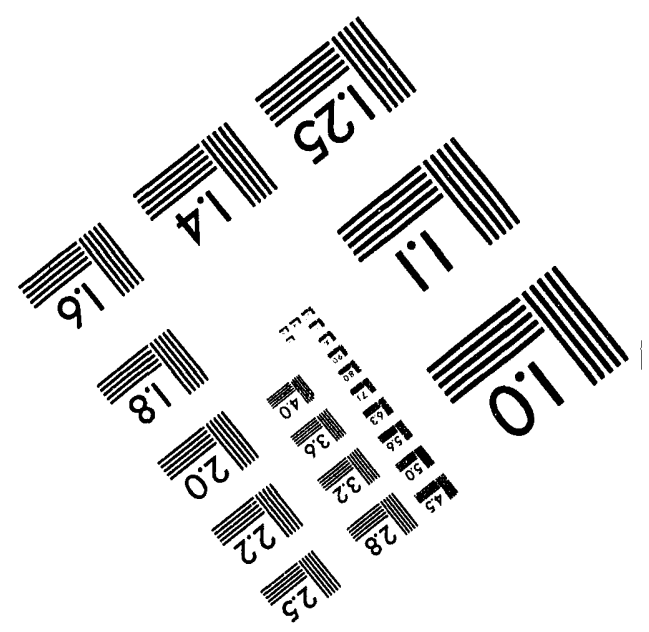



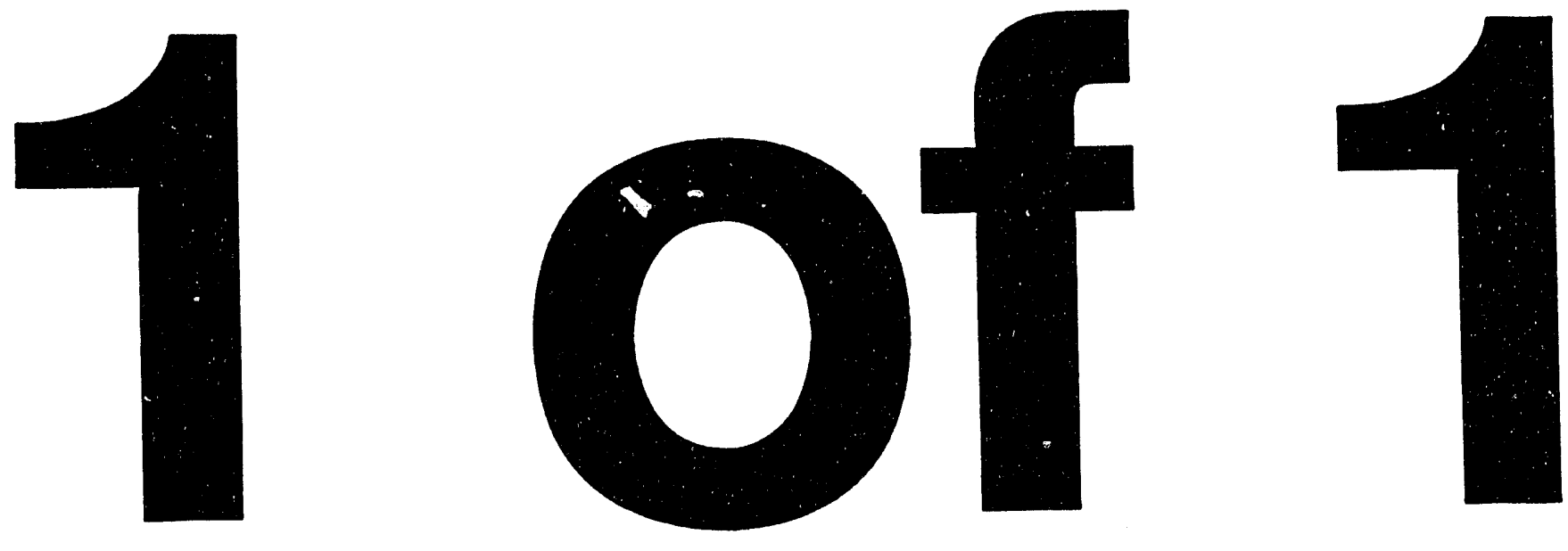
TITLE:

\section{ADAPTIVE CAPTURE OF EXPERT BEHAVIOR}

AUTHOR(S):

Roger D. Jones (CIC-3), Christopher L. Barrett (TSA-DO/SA), Unkyong Hand (TSA-DO/SA), and Ray C. Gordon (TSA-DO)

Sponsor's Report, August, 1994

\section{DISCLAIMER}

This report was prepared as an account of work sponsored by an agency of the United States Government. Neither the United States Government nor any agency thereof, nor any of their employees, makes any warranty, express or implied, or assumes any legal liability or responsibility for the accuracy, completeness, or usefulness of any information, apparatus, product, or process disclosed, or represents that its use would not infringe privately owned rights. Reference herein to any specific commercial product, process, or service by trade name, trademark, manufacturer, or otherwise does not necessarily constitute or imply its endorsement, recommendation, or favoring by the United States Government or any agency thereof. The views and opinions of authors expressed herein do not necessarily state or reflect those of the United States Government or any agency thereof. 


\section{Adaptive Capture of Expert Behavior}

Roger D. Jones, Christopher L. Barrett, Unkyong Hand and Ray C. Gordon

\section{Address}

University of California

Los Alamos National Laboratory

Los Alamos, NM 87545

rdj@lanl.gov

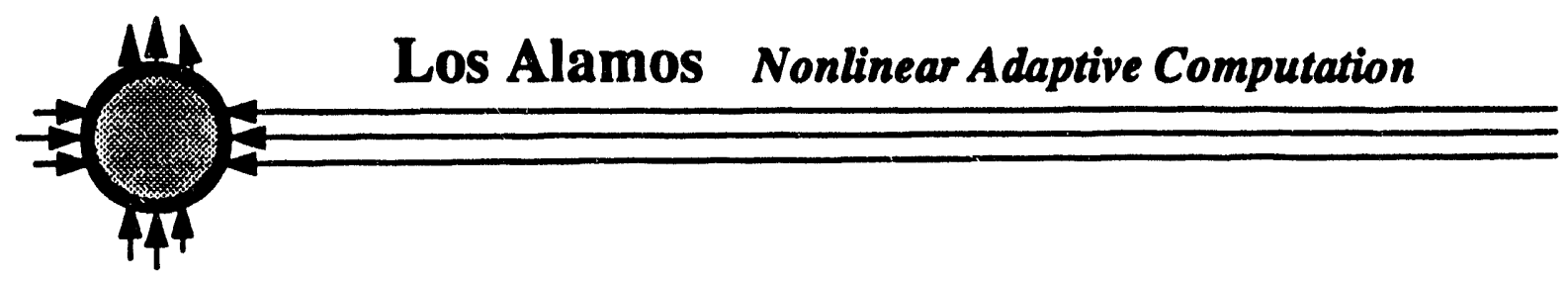

This article is available as a hypertext document, address: http://aws.lanl.gov/neural/ expert_capture/expert_capture.html.

\section{LA-UR-94-}

Los Alamos National Laboratory, an affirmative action/equal opportunity employer, is operated by the University of California for the U.S. Department of Energy under contract W7405-ENG-36. By acceptance of this article, the publisher recognizes that the U.S government retains a nonexclusive, royalty-free license to publish or reproduce the published form of this contribution, or to allow others to do so for U.S. government purposes. The Los Alamos National Laboratory requests that the publisher identify this article as work performed under the auspices of the U.S. Department of Energy.

\section{Abstract}

We smoothed and captured a set of expert rules with adaptive networks. The motivation for doing this is 1) Smoothing leads to stabler control actions. Noise has less effect on the control actions. 2)For some sets of rules, the evaluation of the rules can be sped up. This is important in large-scale simulations where many intelligent elements are present. 3)Variability of the intelligent elements can be achieved by adjusting the weights in an adaptive network. This can be used to mimic human variability. 4)After capture has occurred, the weights can be adjusted based on performance criteria. We thus have the capability of learning a new set of rules that lead to better performance. 
The set of rules we chose to capture were based on a set of threat determining rules for tank commanders. The approach in this paper: 1)We smoothed the rules. The rule set was converted into a simple set of arithmetic statements. Continuous, non-binary inputs, are now permitted. 2)An operational measure of capturability was developed. 3)We chose four candisate networks for the rule set capture: a)Multi-linear network, b)Adaptive Partial Least Squares (PLS) Regression, c)Connectionist Normalized Local Spline (CNLS) Network, and d)CNLS net with a PLS preprocessor. These networks were able to capture the rule set to within a few percent. For the simple tank rule set, the multi-linear network performed the best. When the rules were modified to include more nonlinear behavior, CNLS net performed better than the other three nets which made linear assumptions. 4)The networks were tested for robustness to input noise. Noise levels of plus or minus ten percent had no real effect on the network performance. Noise levels in the plus or minus 30 percent range degraded performance by a factor of two. Some performance enhancement occurred when the networks were trained with noisy data. 5)The scaling of the evaluation time was calculated. The complexity of the rule set in the rule set affects the number of basis functions in the network. 6)Human variation can be mimiced in all the networks by perturbing the weights.

\section{Introduction}

We smoothed and captured a set of expert rules with adaptive networks.

\subsection{Motivation}

The motivation for this work is 1) Smoothing leads to stabler control actions. Noise has less effect on the control actions. 2)For some sets of rules, the evaluation of the rules can be sped up. This is important in large-scale simulations where many intelligent elements are present. 3)Variability of the intelligent elements can be achieved by adjusting the weights in an adaptive network. This can be used to mimic human variability. 4)After capture has occurred, the weights can be adjusted based on performance criteria. We thus have the capability of learning a new set of rules that lead to better performance.

\subsection{Issues}

\subsubsection{Smoothing}

In order to maintain stability in control actions, the rule sets should be smooth rather than binary. For example, a binary rule set might input whether a tank is damaged or undamaged. A smooth rule set would allow for degrees of tank damage.

\section{2 .2 Capturability}

Adaptive elements should be able to reproduce the behavior of a given rule set. The criterion for determining the degree to which this behavior is reproduced should be operationally based. 


\subsubsection{Robustness to Input Noise}

In real situations, one often has incomplete or incorrect state representations. Intelligent elements should be robust to noise in the input state representations.

\subsubsection{Speed}

Adaptive elements can be used as actors in large-scale simulations. When many actors are present, evaluation speed becomes important.

\section{2 .5 Variability}

Often one would like to model the variance in human response in simulation. This can be accomplished by adding noise to the weights in adaptive elements.

\subsubsection{Performance Based Adaptation}

Adaptive elements can replace rule-based elements in simulation. It is then possible to train the individual adaptive elements based on their performance in the large-scale simulation environment. This leads to the identification of a new or improved rule set.

\subsection{Approach}

\section{The Rule Set for Capture}

\section{Rule Smoothing}

The rule set in the previous section is binary in nature. For example, a threat is either damaged or not damaged. There is no prescription for dealing with somewhat damaged threats. A threat is either moving or not moving. How big is the threat if it is barely moving? This binary nature is a common property of many types of expert rule sets.

In this section we generalize the rule set by smoothing the input space. We allow each input variable the be a continuous valued quantity on the interval $[0,1]$. For instance, a partially damaged threat might be assigned an input of 0.345 instead of 0 (damaged) or 1 (undamaged). For convenience, we also map the threat values (TV) onto the interval [0,1], where 1 is the highest threat and 0 is the lowest. The rules for the evaluation of a tank TV given in the previous section can then be succinctly expressed as the arithmetic expression

$$
\tau=\left(1-x_{1}\right)\left[x_{2} x_{3}+0.7 x_{2}\left(1-x_{3}\right)+0.4\left(1-x_{2}\right) x_{3}+0.2\left(1-x_{2}\right)\left(1-x_{3}\right)\right]
$$

\section{Equation 1}

where the TV value is $\tau$, and the inputs are defined by

$$
\begin{aligned}
& x_{1}=0 \text { threat well within range } \\
& x_{1}=1 \text { : threat well out of range }
\end{aligned}
$$




$$
\begin{aligned}
& x_{1}=0.5: \text { threat just within range } \\
& x_{2}=1: \text { threat completely undamaged } \\
& x_{2}=0: \text { threat completely damaged } \\
& x_{3}=1: \text { threat completely still } \\
& x_{3}=0: \text { threat moving rapidly }
\end{aligned}
$$

All intermediate values of the inputs are also permitted.

In rescaling the output, we assumed the minimum TV had value 100 in the expert system rather than 1000 . This allows for a more even population of the output interval with the effect that the leaming systems will be more able to capture the behavior of the rules.

The corresponding smooth rules for the APC TV and the infantry threat are, respectively

$$
\tau=\left(1-x_{1}\right)\left[0.99 x_{2} x_{3}+0.69 x_{2}\left(1-x_{3}\right)+0.39\left(1-x_{2}\right) x_{3}+0.19\left(1-x_{2}\right)\left(1-x_{3}\right)\right]
$$

\section{Equation 2}

and

$$
\tau=\left(1-x_{1}\right)\left[0.98 x_{2} x_{3}+0.68 x_{2}\left(1-x_{3}\right)+0.38\left(1-x_{2}\right) x_{3}+0.18\left(1-x_{2}\right)\left(1-x_{3}\right)\right]
$$

\section{Equation 3}

\section{The Network Architectures}

We will consider four architectures for expert system capture, a multilinear network, Partial Least Squares (PLS)Regression, the Connectionist Normalized Local Spline (CNLS)Network, and a combination of PLS and CNLS. These networks are described in the following sections.

\subsection{The Multilinear Network}

Consider a real valued function $f$ of binary input, $x$. The function can be expressed as a linear combination of $x$ and (1-x). The quantities $x$ and (1-x) can be thought of as two basis furctions which span the binary space and the function $f$ lies within the span. If $f$ is a function of two binary variables, $x_{1}$ and $x_{2}$, then $f$ can be written as a linear combination of $x_{1} x_{2}$, $x_{1}\left(1-x_{2}\right),\left(1-x_{1}\right) x_{2}$, and $\left(1-x_{1}\right)\left(1-x_{2}\right)$. If $\mathrm{f}$ is a function of three inputs there are correspondingly eight basis functions which span the space. They are $x_{1} x_{2} x_{3}$. 


$$
\begin{aligned}
& x_{1} x_{2}\left(1-x_{3}\right), x_{1}\left(1-x_{2}\right) x_{3}, x_{1}\left(1-x_{2}\right)\left(1-x_{3}\right),\left(1-x_{1}\right) x_{2} x_{3}, \\
& \left(1-x_{1}\right) x_{2}\left(1-x_{3}\right),\left(1-x_{1}\right)\left(1-x_{2}\right) x_{3} \text { and }\left(1-x_{1}\right)\left(1-x_{2}\right)\left(1-x_{3}\right) .
\end{aligned}
$$

If the inputs are real, these basis functions no longer span the space. For example the function $f(x)=x^{2}: x$ real

cannot be captured by these basis functions. The smoothed rules of the previous section, however, fall within the span of this basis set. Equations 1-3, in fact, are explicit linear combinations of the basis functions of this section.

We therefore construct an architecture

$$
\phi(t)=\sum_{j=1}^{N} c_{j} u_{j}(x)
$$

\section{Equation 4}

to capture the smoothed rules. Here, the u's are the basis functions given above, the c's are adjustable coefficients, and $\mathrm{N}$ is the total number of basis functions. The output of the network, $\phi$, is matched to the output, $\tau$, by adjusting or training the coefficients, $c$.

The training algorithm is given by

$$
c_{j}^{t+1}=c_{j}^{t}+\eta\left(\tau_{t}-\phi\left(x_{t}\right)\right) \frac{u_{j}\left(x_{t}\right)}{\sum_{k=1}^{N} u_{k}^{2}\left(x_{t}\right)}
$$

\section{Equation 5}

where $\eta$ is a learning rate and the superscript(subscript) $t$ indexes a presentation of training data to the network.

It is not difficult to come up with a rule that this architecture cannot capture. An example would be, "Ignore threat if it is either too close or out of range. Otherwise TV is very large." This rule cannot be expressed with a binary input. For this reason we consider a more general class of architectures in the following sections.

\subsection{Partial Least Squares Regression (PLSR)}

Partial Least Squares (PLS) is a generalization of Principle Component Analysis (PCA). PCA is an algorithm which identifies those directions in input space in which the strongest correlations exist. PLS, on the other hand, is an algorithm which identifies those directions in input space which are most strongly correlated with output. PLS, like PCA, can be used to reduce the input dimension of a problem. Since output is taken into account, the number of 
effective inputs generated by PLS is often much smaller than the number of effective inputs generated by PCA. Nonadaptive versions of these algorithms are presented in the monograph by Martens and Naes [25]. We present here an adaptive version of the PLS algorithm.

1. Scale input variables and output variable (assume scalar output) so they are all order one.

2. Find the current average in the input.

$$
\begin{aligned}
& \langle x\rangle^{(t+1)}=\langle x\rangle^{t}+\eta\left(t^{t}-\langle t\rangle^{t}\right) \\
& \langle y\rangle^{(t+1)}=\langle y\rangle^{t}+\eta\left(y^{t}-\langle y\rangle^{t}\right)
\end{aligned}
$$

Here, $\eta$ is a leaming rate and $t$ is an index which identifies the training point.

3. Center data.

$$
\begin{aligned}
& t_{0}=t-\langle t\rangle \\
& y_{0}=y-\langle y\rangle
\end{aligned}
$$

The subscript $\mathbf{0}$ indexes the iteration. Each iteration is associated with an input vector correlated with the output. Succeeding iterations are successively less significant.

4. Find the vector $\ggg$ in input space most strongly correlated with the output.

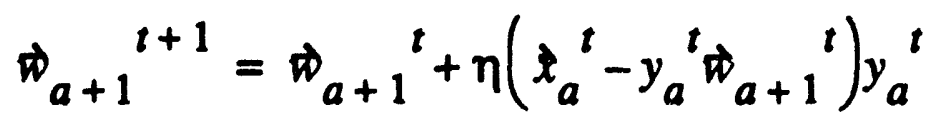

5. Scale the vector to length one.

$\varpi_{a+1} \cdot \pitchfork_{a+1}=1$

6. Calculate "scores" t.

$$
t_{a}=\not_{a+1} \cdot t_{a}
$$

7. Calculate "spectral loadings"

$$
\vec{p}_{a+1}{ }^{t+1}=\dot{p}_{a+1}{ }^{t}+\eta\left(t_{a}^{t}-t_{a}^{t} \hat{p}_{a+1}{ }^{t}\right) t_{a}^{t}
$$

8. Calculate "chemical loading"

$$
q_{a+1}{ }^{t+1}=q_{a+1}{ }^{t}+\eta\left(y_{a}^{t}-t_{a}^{t} q_{a+1}{ }^{t}\right) t_{a}^{t}
$$

9. Update $x$ and $y$. 


$$
\begin{aligned}
& t_{a+1}=t_{a}-t_{a} p_{a+1} \\
& y_{a+1}=y_{a}-t_{a} q_{a+1} \\
& a \Rightarrow a+1
\end{aligned}
$$

10. If $a$ is less than $A$, the total desired number of cardinal components then go to 4 .

After $A$ iterations the output is given by

$y=\langle y\rangle+t_{1} q_{1}+t_{2} q_{2}+t_{3} q_{3}+\ldots+t_{A} q_{A}$

The procedure we use for regression is presented in Fig. 1. A training file consisting of input/ output examples is used to train the loadings using the adaptive PLS scheme. This is represented by the boxes on the left. A test file, with new data is run through the PLS algorithm with the loadings that were generated by the training file. This generates an estimate of the output ypls for each input from the testing file.

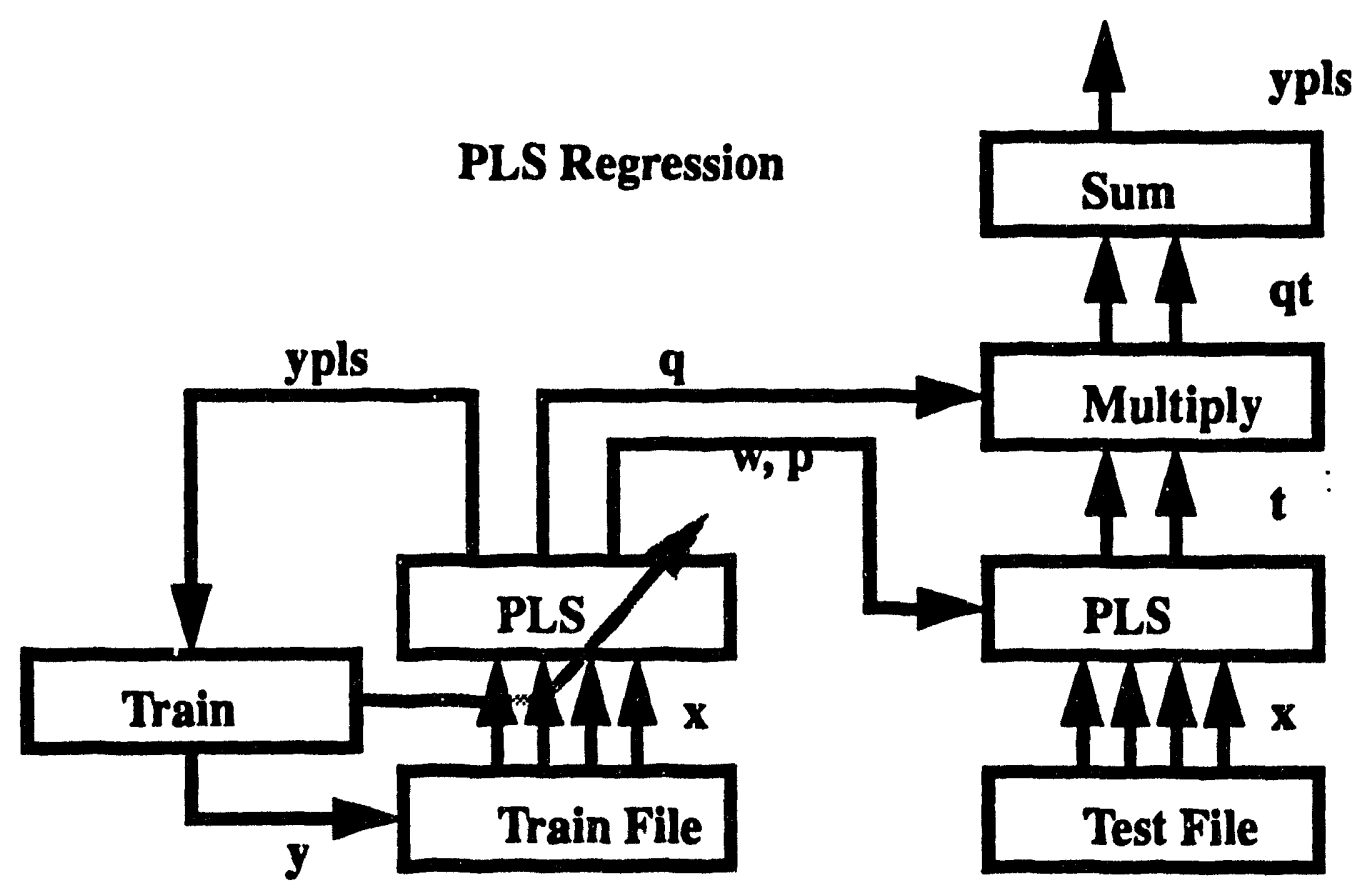

Figure 1. Block diagram of adaptive PLS regression algorithm. 


\subsection{The Connectionist Normalized Local Spline (CNLS) Network}

\subsubsection{The Architecture}

The Connectionist Normalized Local Spline (CNLS) network is an architecture that improves interpolation and reduces the amount of training as compared with Multi-layer Perceptrons.[1,9-12] The architecture can be obtained from the identity:

$$
g(t)=\frac{\sum_{j=1}^{N} g(t) \rho_{j}(t)}{\sum_{j=1}^{N} \rho_{j}(t)}
$$

Here, $p_{j}(t)$ is a localized function of $t$ about some $t$. Hence, $g(t)$ on the right of Equation. 6 can be approximated by its Taylor expansion about $\vec{d}$. We have then,

$$
\phi(t)=\sum_{j=1}^{N}\left[f_{j}+\left(t-\grave{a}_{j}\right) \cdot \vec{a}_{j}\right] \frac{\rho_{j}(t)}{\sum_{k=1}^{N} \rho_{k}(t)}
$$

Equation 7

for an approximation to $g(t)$. We regard the quantities $f$ and $d$ as adjustable parameters. Equation 78 defines the CNLS net architecture. This net differs from standard radial basis function nets in two ways, the use of normalization and also a linear term, $\left(t-\vec{a}_{j}\right) \cdot \vec{a}_{j}$. The addition of these two terms is responsible for the reduction in the amount of training data needed to obtain reasonable approximations. As in the case with radial basis functions, the training of $f_{j}$ and $\vec{a}_{j}$ is linear and hence very fast. The widths of the basis functions can also be trained. This training is nonlinear. The locations of the basis function centers can also be trained.

\subsubsection{The Learning Algorithms}

The training for the linear quantities, $f_{j}$ and $\vec{d}_{j}$ is given by 


$$
f_{j}^{t+1}=f_{j}^{t}+\eta\left[g\left(t_{t}\right)-\phi\left(t_{t}\right)\right] \frac{\rho_{j}\left(t_{t}\right) \sum_{j=1}^{N} \rho_{j}\left(t_{t}\right)}{\sum_{j=1}^{N}\left[\rho^{2}{ }_{j}\left(t_{t}\right)+\beta_{j}\left(t_{t}-t_{j}\right)^{2} \rho_{j}{ }^{2}\left(t_{t}\right)\right]}
$$

Equation 89

and

$$
a_{j}^{t+1}=a_{j}^{t}+\eta\left[g\left(t_{t}\right)-\phi\left(t_{t}\right)\right] \frac{\rho_{j}\left(t_{t}\right) \beta_{j}\left(t_{t}-a_{j}\right) \sum_{j=1}^{N} \rho_{j}\left(t_{t}\right)}{\sum_{j=1}^{N}\left[\rho^{2}{ }_{j}\left(t_{t}\right)+\beta_{j}\left(t_{t}-\bar{a}_{j}\right)^{2} \rho_{j}{ }^{2}\left(t_{t}\right)\right]}
$$

\section{Equation 9}

Here, $t_{t}$ is a training input vector and $g\left(t_{t}\right)$ is the training output for the $t^{\prime}$ th on-line presentation of data to the network. The superscripts $t$ and $t+1$ indicate values of the weights before and after the $t^{\prime}$ th presentation of training data. The learning rate, $\eta$, is the leaming rate. The quantity $\beta_{j}$ is the inverse square width of the $j^{\prime}$ th basis function.

\subsubsection{Form of the Basis Functions}

The basis functions are usually taken to be unnormalized gaussians

$$
\rho_{j}(t)=\exp \left[-\beta_{j}\left(t-\bar{a}_{j}\right)^{2}\right] \text {. }
$$

\subsection{CNLS Net With a PLS Preprocessor}

If the input space has high dimensionality, the CNLS net tends to fail. In that situation, the PLS algorithm can be used as a preprocessor to the CNLS net. The PLS algorithm can reduce the dimensionality while the CNLS algorithm can capture nonlinearities. In this situation we use either the score, $t$, or the weighted scores, qt, as inputs to the CNLS net. Training for the PLS algorithm and for the CNLS algorithm can occur simultaneously.

\subsection{Multilinear Net with PLS Postprocessor}

The PLS algorithm can be used to reduce the number of basis functions in the network. This is important when the evaluation speed of the network is important. When the rule set of Equations 1-3 are captured by a multi-linear net, for example, there are many terms in the net 
with zero coefficients. The PLS processor can be used to prune these terms. This has no little effect on the accuracy of the net, so we will not explicitly consider this net in accuracy performance measurements.

\section{Capturability}

In this section we define a criterion for measuring the capturability of the network and compare the various network performances. We find that all the networks are able to capture the rule set to within 2 percent. Since the rule set falls within the span of the multi-linear network, this neiwork is able to capture the rule set to round-off error. When the input rules are modified so that they no longer lie within the span of the multi-linear network, then that network's performance is comparable to the others.

\subsection{Criterion for Capture}

The output of each of the networks is a TV for each of the threats. The TV as determined by the rule based system is given by Equations 1-3. The TV is actually a subjective value. The key operational quantity is the rank ordering of the threats. Any systems with the same rank ordering, even if the actual values for the TV's are different, are operationally equivalent. This allows us to specify a performance criterion:

$$
R=\frac{1}{T} \sum_{\substack{j=1 \\ \text { Equation } 10}}^{l} \tau_{j}
$$

where

$$
T=\sum_{j=1}^{L} \tau_{j}
$$

\section{Equation 11}

Here, $L$ is the total number of threats and the performance value, $R$, is called the risk. The summation in Equation 10 is over 1 values of $\tau$. The values are chosen as the 1 largest TV's as determined by a rank ordering. This rank ordering is obtained from Equations 1-3 or from estimates of the TV's as determined by a network. The actual values of the TV's as determined by Equations 1-3 are used in the summation rather than estimates obtained from the network.

The risk as calculated from the TV's of Equations 1-3 will be called the ground truth risk. The ground truth risk is an upper limit on the risk value for each 1 . To see this note that if the network captures the ranking exactly, the risk calculated from that ranking will equal the ground truth risk. If, however, the ranking is different for the network than the ground truth ranking, then a high value TV within the summation set will be substituted for a lower ranking TV. The net effect will be to lower the value of the summation. Ground truth risk for 
tank threats is plotted in Figure 2a. The TV's were calcuiated by choosing random values for the inputs, $x$. There were 800 threats in all. The difference between this risk and the risk calculated from the network is the measure of performance.

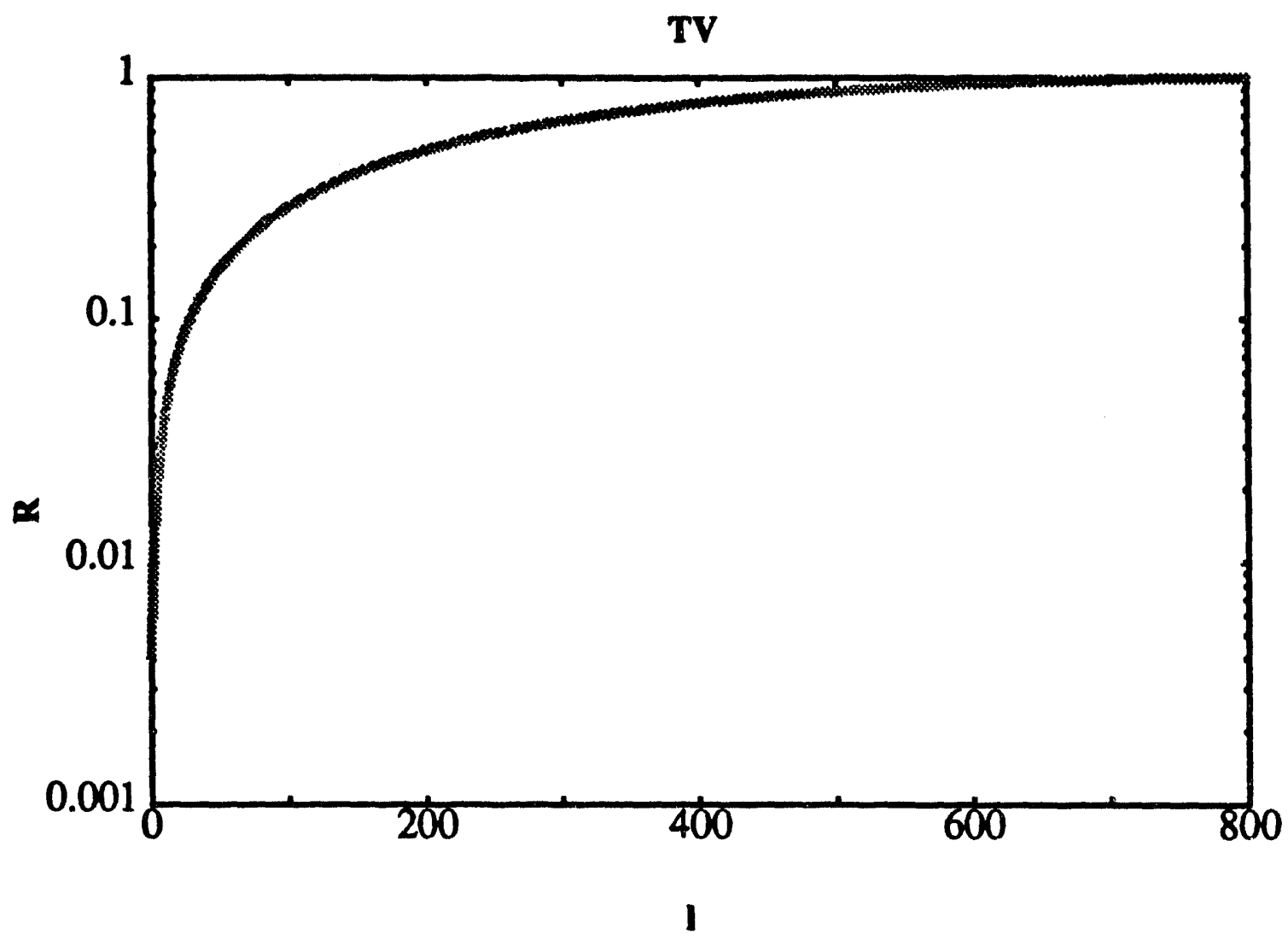

Figure 2a. Peiformance Criterion for Capturability

\subsection{Network Performance on Smooth Rule Set}

The networks were trained on a set composed of 800 threats chosen by randomly picking input values between 0 and 1 . The data were shown to the networks 20 times. All learning rates were taken to be 0.01 . The values of $\beta$ were chosen to be 10 for the CNLS network and 100 for the CNLS network with the PLS preprocessor. The results are not sensitive to the choice of $\beta$. The basis function centers for the CNLS nets were chosen to lie on the comers of the unit hypercube. Since there were three input dimensions, there were eight basis functions in all. The test set was composed of 800 patterns chosen in the same manner as the training set.

The results are displayed in Figure 3. Here we plot the difference between ground truth risk and risk as calculated by the network. The PLS outperforms CNLS for small values of 1 . For large values, however, the situation is reversed. The CNLS with PLS preprocessor captures 
the better performance of PLS and CNLS. For small values of 1, PLS-CNLS is comparable to PLS. For large values, PLS-CNLS is comparable to CNLS. The best performer by far on this data set, however, was the multi-linear net. This network learned the ground truth to round-off error. This occurred because Equation 1 lies within the span of the multi-linear network (it is of the same functional form). These results are also plotted in Figure 2. Since the fits are so good, the differences are not visible in this plot.

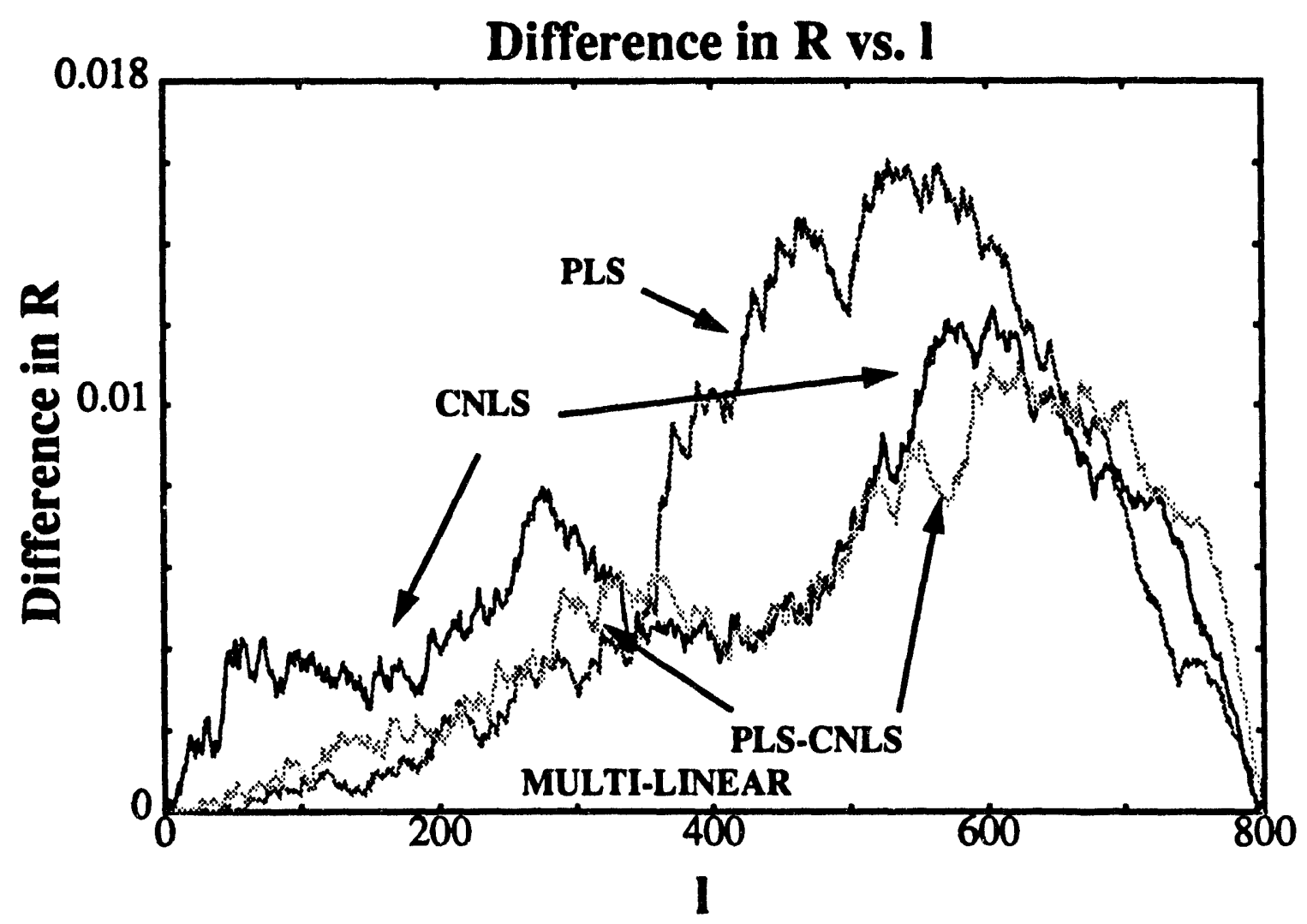

Figure 3. Difference in $R$ vs. I for simple rule set.

The rule set we have chosen to capture is very simple. The behavior is multi-linear. This is a consequence of the binary nature of the design. For a more complex rule set, the behavior of the networks is much different. Suppose we replace $x_{1}$ in Equation 1 with $4.0 x_{1}\left(1-x_{1}\right)$.

This destroys many of the linear characteristics of the rules. A rule that might generate this term could be "ignore threat if it is too far or too near." Figure 4 displays the performance for this rule set. As expected the nets that rely on linear behavior, PLS, PLS-CNLS, and Multilinear, perform poorly. CNLS net, on the other hand still captures the rule base to within 5 percent. 


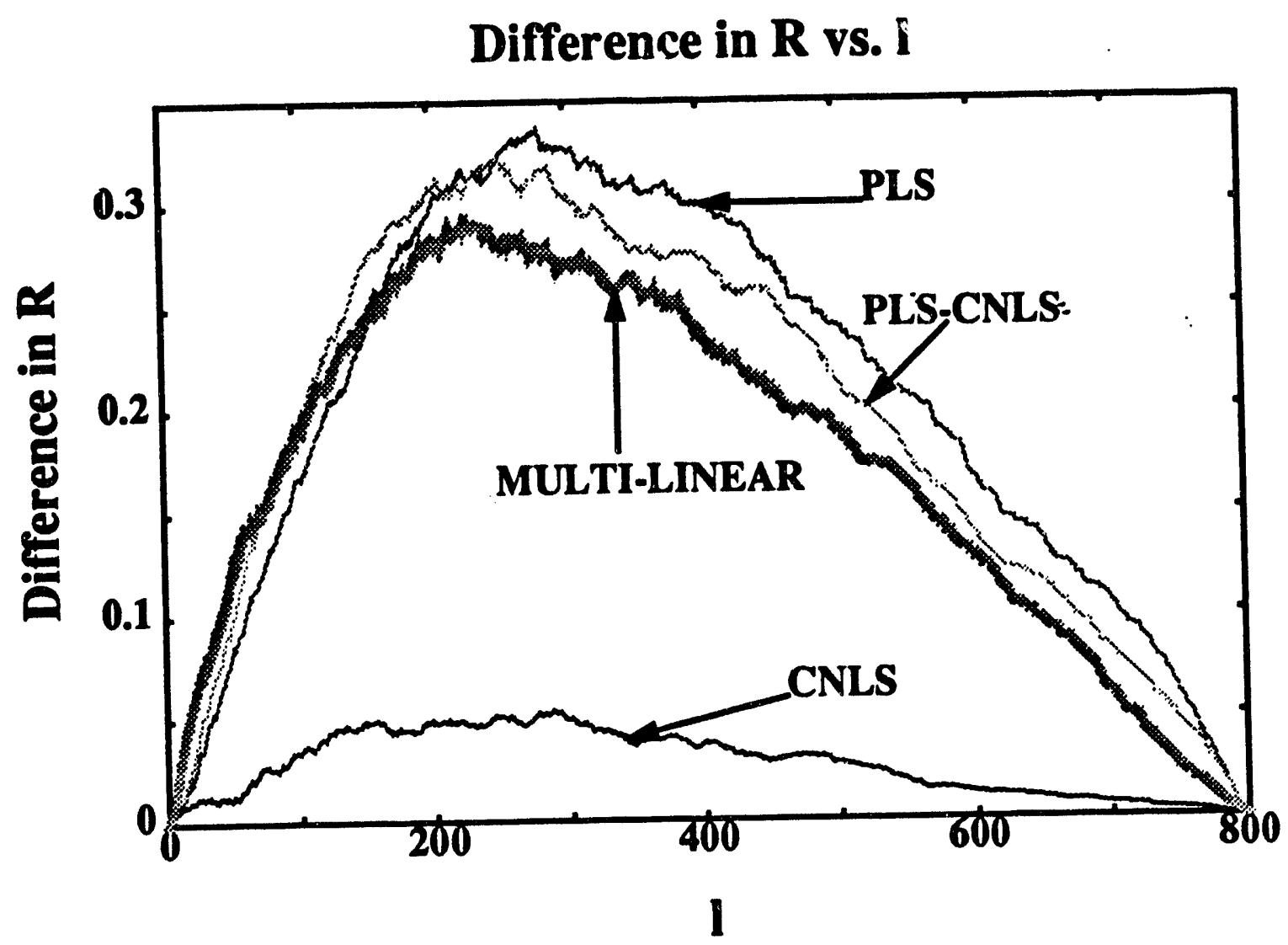

Figure 4. Difference in $R$ vs. If for a quadratic rule set.

\subsection{Comparison of Binary and Smooth Rule Sets}

It is interesting to compare the risks for the smooth rules of Equation 1 and the same rule set but with binary inputs. To generate the binary rules, we thresholded the random inputs. If an input was greater than 0.5 , we changed it to one. If it was less than 0.5 , we changed it to zero. The rank ordering for the smooth and the binary rule set are displayed in Figure $2 \mathrm{~b}$. Each curve was calculated as if it were ground truth. There is some difference in the ordering between the two rule sets. External validation is required to determine which is the preferable rule set. This can be done by examining the performance of the two rule sets in simulation.

The networks captured the binary rule set exactly. There are effectively eight unique data points in the training set and each network has at least eight adjustable parameters. The binary behavior is therefore exactly capturable. 


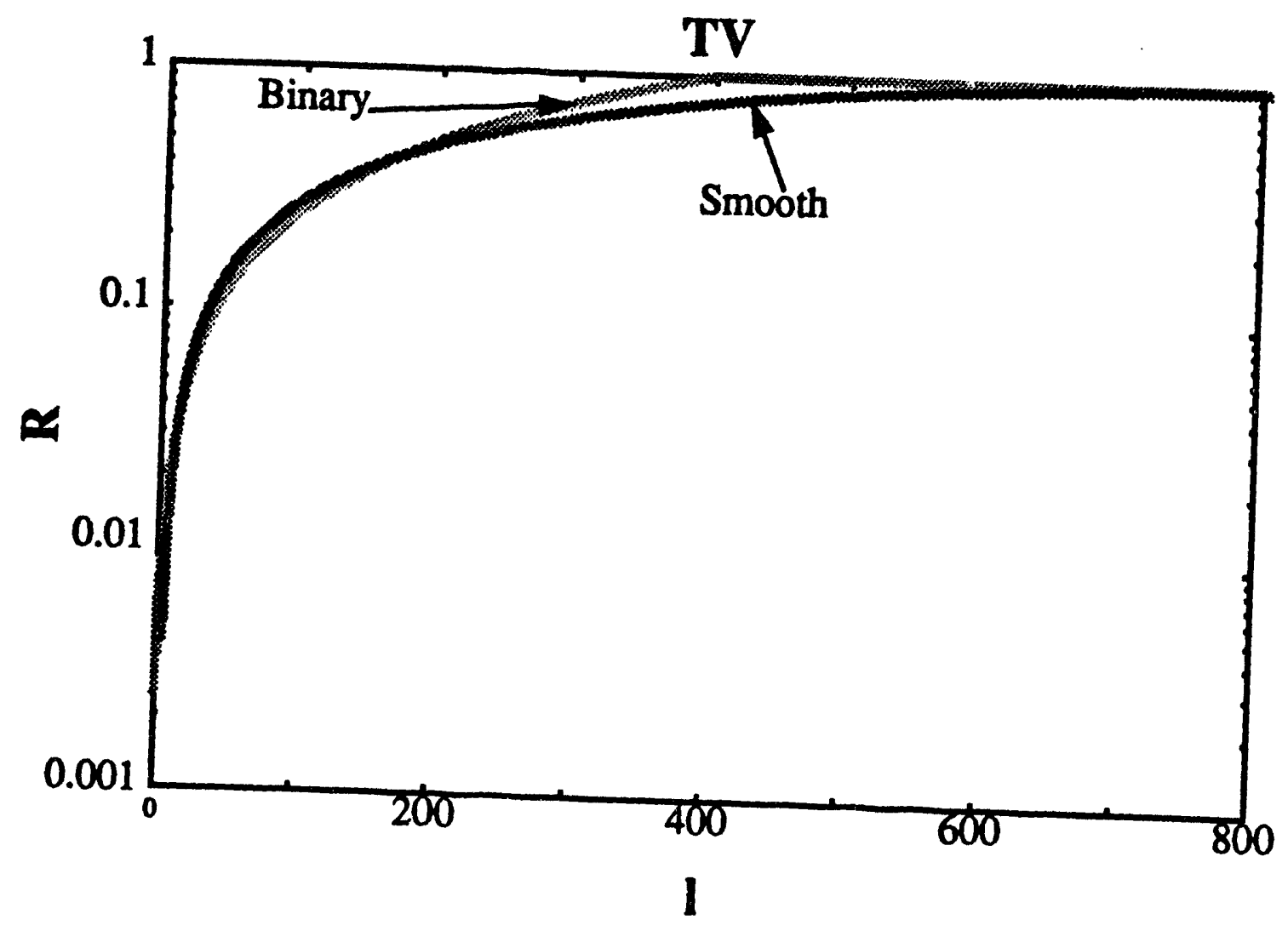

Figure 2b. Comparison of risk for smooth and binary rules

\section{Robustness}

Quite often there is noise in the input signal. The actual state of a system is unclear. In this
section we examine the effect of noisy inputs on netwot perfoll section we examine the effect of noisy inputs on network performance. The noise in the
inputs will be modeled as

\section{$t \rightarrow t \pm a \varepsilon$}

\section{Equation 12}

where $\varepsilon$ is a random vector with components between zero and one and a is the noise amplitude. We will first consider noise only in the test inputs. The values of the inputs are not constrained to lie in the interval $[0,1]$. We assume perfect information for training. We will then demonstrate that some performance gains can be seen by training with noisy inputs.

Performance levels with a noise amplitude of 0.1 are displayed in Figure 5. Since there is no noise in training and the multi-linear network is able to capture the rule set to roundoff, the 
curve marked MULTI-I INEAR also captures the response of the rule set to input noise. At this noise level there is no significant degradation in performance of the PLS, CNLS, and PLSCNLS networks over their performance illustrated in Figure 3.

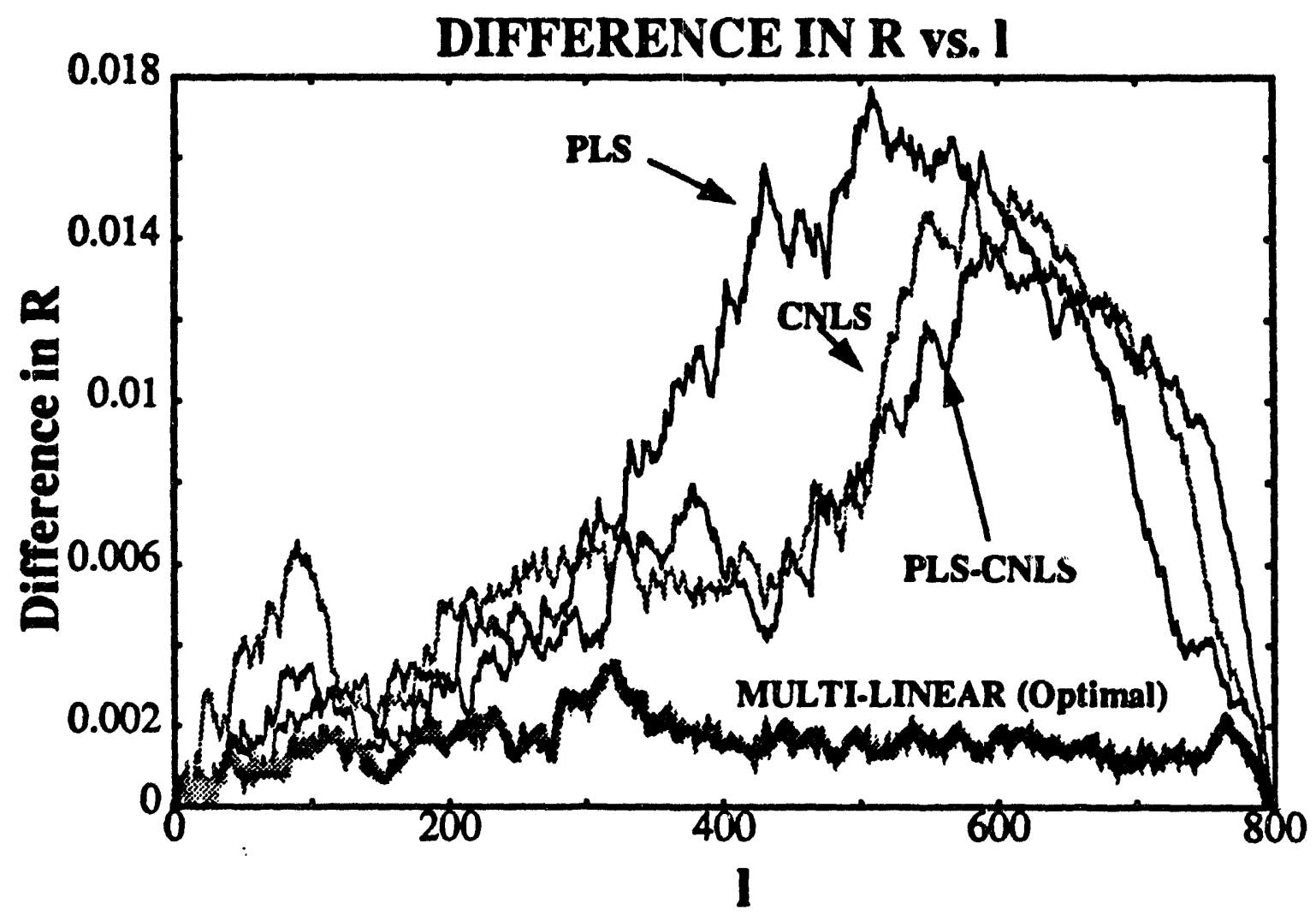

Figure 5. Network performance for input noise levels of plus or minus 10 percent. Note the degraded performance of the multi-linear network as compared with the performance displayed in Figure 2. Since the multi-linear network learned the rule to roundoff, this represents optimal performance. The other networks show no serious degradation over the noise free case.

In Figure 6 we increase the noise level to an amplitude of 0.30 . We see here an approximately 50 percent degradation in performance of the PLS, CNLS, and PLS-CNLS nets. For low 1 the performance is near optimal, as can be seen in a comparison with the MULTI-LINEAR net. For large 1 , the performance deteriorates. 


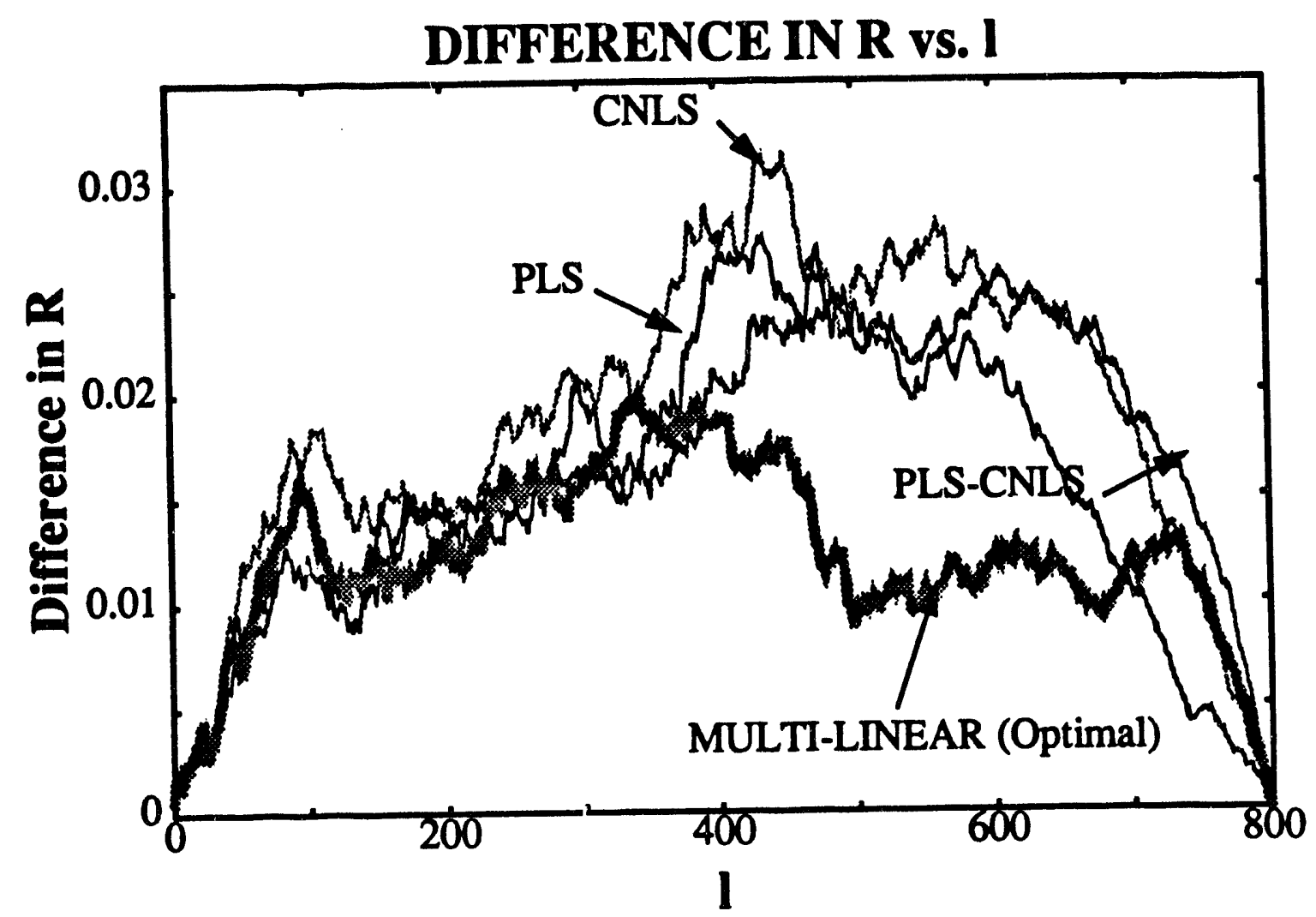

Figure 6. Noise level at plus or minus 30 percent. There is nearly a factor of two degradation in performance over the noise free case.

For a noise amplitude of 0.6 the re is approximately a factor of five degradation in performance. 


\section{DIFFERENCE IN R vS. I}

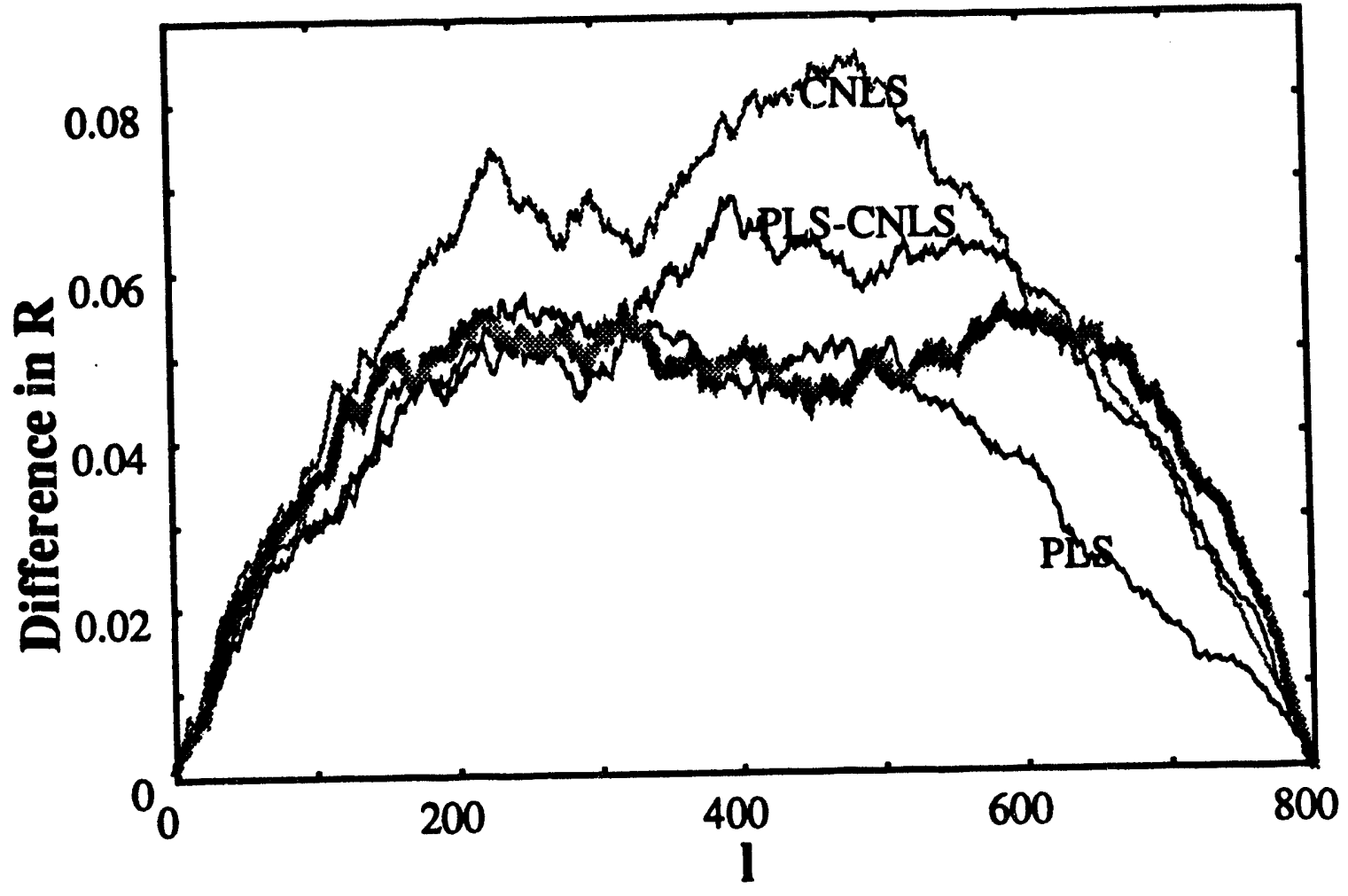

Figure 7. Noise level at plus or minus 60 percent.

If the system is tested with noisy inputs one might expect that a performance enhancement can be obtained if the networks are trained with comparable noise levels. One, in fact, sees a small improvement when this is done. This is illustrated in Figure 8. This figure should be compared with Figure 6. 


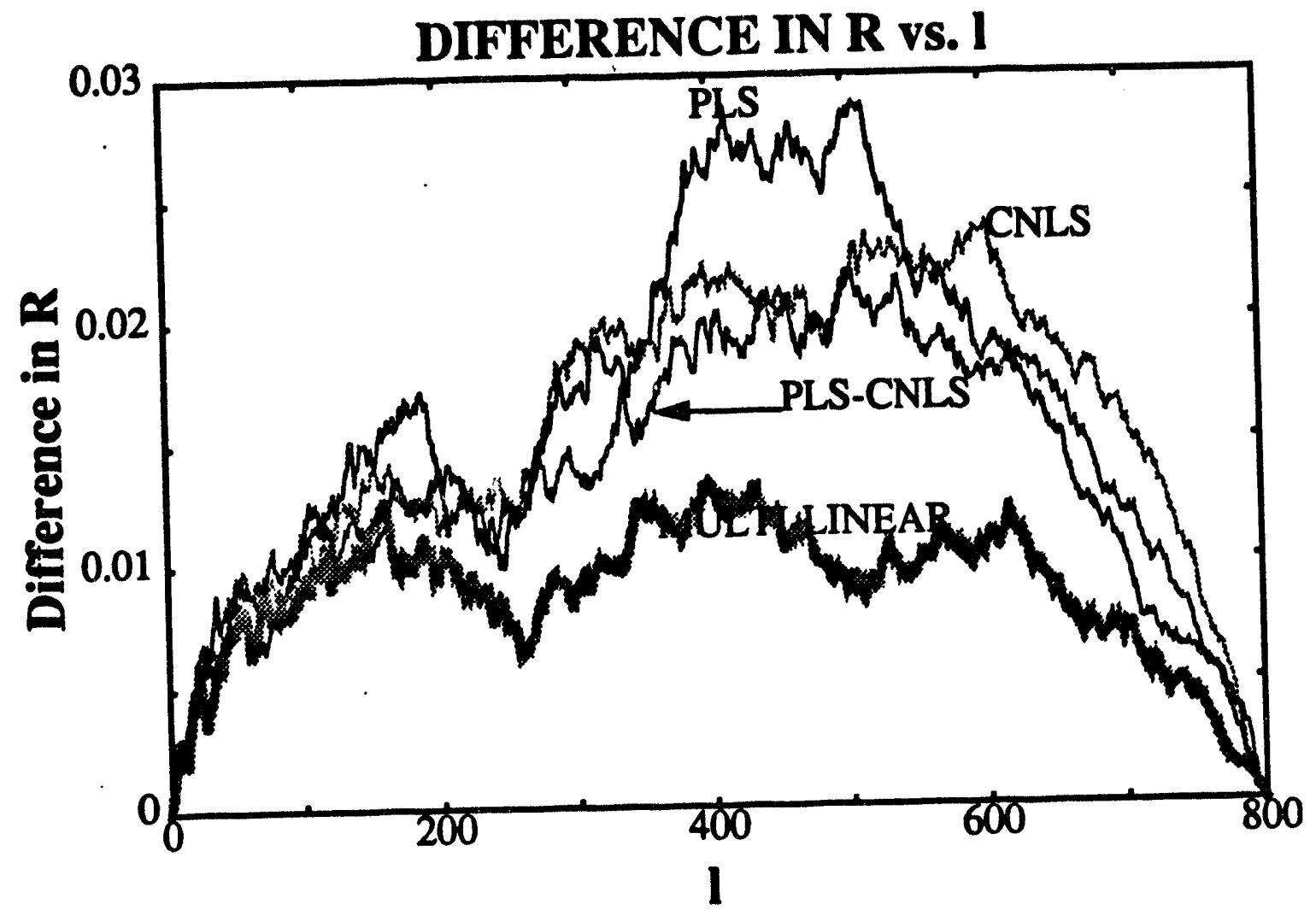

Figure 8. Performance with training on noisy inputs. The noise amplitude is 0.3 . One can see a small improvement in performance when compared with Figure 6.

\section{Speed}

Networks that capture rule sets can be used as intelligent elements in large scale simulations. In this application speed is an important issue. The networks need to be computationally as fast or faster than the underlying rule set. Of the networks considered here, the multi-linear network and the Partial Least Squares are the fastest. If the rule set is simple enough to allow capture by this network then the multi-linear network is the obvious choice. The time for a single evaluation of the network is

$t_{m l}=\dot{B I}\left(m+\frac{1}{2} a\right)$

\section{Equation 13}

where $m$ is time for a multiply, $a$ is the time for an addition, $I$ is the input dimension, and $B$ is the number of basis functions. The number of basis functions without postprocessing is

$$
B=2^{I}
$$




\section{Equation 14}

If we use a PLS postprocessor then we can significantly reduce the number of basis functions for simple rule sets. For example, take the extreme case. Suppose the rule set was to always output one no matter what the input. The number of basis functions needed to represent this rule is one, the constant one. The input dimension in Equation 14 would effectively be zero. With this analogy we can write the corresponding relationship for Equation 14 when postprocessing occurs as

$B=2^{I^{*}}$

\section{Equarion 15}

where $I^{*}$ is the effective complexity dimension of the rule set. In real situations this dimension is often difficult to calculate. As a rule of thumb, for many realistic rule sets, Equations 14-15 generate a large upper limit on the net evaluation time.

The other networks scale similarly to Equation 13. The differences are in the numerical coefficients of $m$ and $a$ that tend to increase the evaluation time.

\section{Variability}

In large-scale simulations one often would like to mimic human variability in the execution of the rule sets. This can easily be accomplished by varying the weights in the networks. In the multi-linear network, for instance, each coefficient weights the strength of a specific rule in the rule set. These weights can be manipulated manually or randomly. The weights in the other networks have less physical meaning. their manipulation, however, yields results similar to the manipulation of the multi-linear network.

\section{Concluding Remarks}

Our goal was to smooth and capture a set of expert rules with adaptive networks. There are a number of reasons for doing this:

- Smoothing leads to stabler control actions. Noise has less effect on the control actions.

- For some sets of rules, the evaluation of the rules can be sped up. This is important in large-scale simulations where many intelligent elements are present.

- Variability of the intelligent elements can be achieved by adjusting the weights in an adaptive network. This can be used to mimic human variability.

- After capture has occurred, the weights can be adjusted based on performance criteria. We thus have the capability of learning a new set of rules that lead to better performance.

The set of rules we chose to capture were based on a set of threat determining rules for tank commanders. The approach in this paper:

- We smoothed the rules. The outcome is illustrated in Equations 1-3. The rule set was converted into a simple set of arithmetic statements. Continuous, non-binary inputs, are now permitted. 
- An operational measure of capturability was developed.

- We chose four candidate networks for the rule set capture:

- Mult-linear network. This network is a multi-linear combination of the inputs. This network spans the space of the smoothed rule set. It was able to capture the rule set to roundoff error. This network, however, does not have an advantage over other networks for more complex rule sets.

- Adaptive Partial Least Squares (PLS) Regression. This is a linear method related to Principle Component Analysis. It finds the directions in input space most correlated with the output. Since it is linear, it is very fast.

- Connectionist Normalized Local Spline (CNLS) Network. The is a generalized local radial basis function network. It is well suited to the capture of nonlinear rules in low dimensional spaces.

- CNLS net with a PLS preprocessor. This net is designed for rule capture in high dimensional spaces.

These networks were able to capture the rule set to within a few percent. For the simple tank rule set, the multi-linear network performed the best. When the rules were modified to include more nonlinear behavior, CNLS net performed better than the other three nets which made linear assumptions.

- The networks were tested for robustness to input noise. Noise levels of plus or minus ten percent had no real effect on the network performance. Noise levels in the plus or minus 30 percent range degraded performance by a factor of two. Some performance enhancement occurred when the networks were trained with noisy data.

- The scaling of the evaluation time is given by Equation 13. The complexity of the rule set in the rule set affects the number of basis functions in the network.

- Human variation can be mimiced in all the networks by perturbing the weights.

\section{Acknowledgments}

\section{References and Bibliography}

[1] P. J. Werbos, "Beyond regression: New rools for prediction and analysis in the behavioral sciences." Masters thesis, Harvard University. (1974); D. B. Parker, "Leaming logic." Invention Report S81-64, File 1, Office of Technology Licensing, Stanford University, Stanford, CA. (1982); D. E. Rumelhart, G. E. Hinton, and R. J. Williams, Leaming internal representations by error propagation. in Parallel Distributed Processing, eds. D. E. Rumelhart and J. L. McClelland, 1 (1986) 318-362.

[2] W. H. Press, B. P. Flannery, S. A. Teukolsky, and W. T. Vetterling, Numerical Recipes, (Cambridge University Press, Cambridge, 1986)

[3] A. S. Lapedes and R. Farber, "Nonlinear signal processing using neural networks: Prediction and system modeling." Technical Report, Los Alamos National Laboratory, Los Alamos, New Mexico 87545 (1987).

[4] A. S. Lapedes and R. Farber, "How neural nets work." in Neural Information Processing Systems, ed. D. Z. Anderson, (AIP, New York, 1988) 442-456. 
[5] D. Nguyen, and B. Widrow. "The truck backer-upper: An example of self-learning in neural networks." Proceedings IJCNN, Washington (June 1989), (1989) pp. II-357-363.

[6] J. D. Farmer and J. J. Sidorovich, "Predicting chaotic time series," Physical Review Letters, 59, (1987) 845.

[7] J. Moody and C. J. Darken, "Fast learning in networks of locally tuned processing units," Neural Computation, 1, (1989) 281-294.

[8] M. Casdagli, "Nonlinear prediction of chaotic time series," Physica D, 35, (1989) 335356.

[9] Y. C. Lee, "Neural networks with memory for intelligent computations," in Proceedings of the 13th Conference on the Numerical Simulation of Plasmas. Santa Fe, New Mexico, September 17-20, 1989. (1989).

[10] R. D. Jones, Y. C. Lee, C. W. Barnes, G. W. Flake, K. Lee, P. S. Lewis and S. Qian, "Function approximation and time series prediction with neural networks," in Proceedings of the International Joint Conference on Neural Networks, San Diego, California, June 17-21, 1990. (1990) 1-649-666.

[11] J. A. Howell, C. W. Barnes, S. K. Brown, G. W. Flake, R. D. Jones, Y. C. Lee, S. Qian, and R.M. Wright, "Control of a negative-ion accelerator source using neural networks," Proceedings of the International Conference on Accelerator and Large Experimental Physics Control Systems Vancouver, B.C., Canada October 30-November 3, 1989. Los Alamos report LA-UR-89-3597. (1989).

[12] R. D. Jones, C. W. Barnes, G. W. Flake, K. Lee, Y. C. Lee, P. S. Lewis, M. K. O'Rourke and S. Qian, "Prediction and Control of Chaotic Processes Using Nonlinear Adaptive Networks," in Proceedings for Nonlinear and Chaotic Processes in Plasmas, Fluids, and Solids, July 23-27, 1990, Edmonton, Alberta, Canada. (1990).

[13] R. C. Davidson, Methods of Nonlinear Plasma Theory, (Academic Press, New York, 1972), pp. 15-18.

[14] M. Barnsley, Fractals Everywhere, (Academic, San Diego, 1988).

[15] M. D. Davis and E. J. Weyuker, Computability, Complexity, and Languages: Fundamenzals of Theoretical Computer Science, (Academic, San Diego, 1983).

[16] S. Wolfram, Theory and Applications of Cellular Automata, (World Scientific, Singapore, 1986).

[17] A. R. Smith, "Simple computational-universal cellular spaces and self-reproduction," in IConference Record, IEEE 9th Annual Symposium on switching and automata theory, (1968) 269-277; J. W. Thatcher, "Universality in the von Neumann cellular model," in Essays on cellular automata, ed. A. W. Burks, (University of Illinois Press, 1970) pp. 132-186; E. F. Codd, "Propagation, computation and construction in two-dimensional cellular spaces," in Cellular Automata, (Academic, New York, 1965); J. von Neumann, "The general and logical theory of automata," in Cerebral Mechanisms in Behavior: The Hixon Symposium, (John Wiley and Sons, 1951). 
[18] Y. C. Lee, S. Qian, R. D. Jones, C. W. Barnes, G. W. Flake, M. K. O'Rourke, K. Lee, H. H. Chen, G. Z. Sun, Y. Q. Zhang, D. Chen, and G. L. Giles, "Adaptive stochastic cellular automata: theory," Physica D 45 (1990) 159.

[19] S. Qian, Y. C. Lee, R. D. Jones, C. W. Barnes, G. W. Flakr M. K. O'Rourke, K. Lee, H. H. Chen, G. Z. Sun, Y. Q. Zhang, D. Chen, and G. L. Giles, "Adaptive stochastic cellular automata: applications," Physica D 45 (1990) 181.

[20] A. G. Barto, R. S. Sutton, and C. W. Anderson, "Neuronlike adaptive elements that can solve difficult learning control problems," IEEE Transactions on Systems, Man, and Cybernetics SMC-13 (1983) pp. 834-846.

[21] S. Qian, Y. C. Lee, R. D. Jones, C. W. Barnes, and K. Lee "Function approximation with an orthogonal basis net," in Proceedings of the International Joint Conference on Neural Networks, San Diego, California, June 17-21, 1990. (1990) III-605-620.

[22] M. C. Mackey, and L. Glass, "Oscillation and chaos in physiological control systems", Science 197 (1977) 287.

[23] A. Gelb (ed.), Applied Optimal Estimation, (MIT Press, Cambridge, 1974).

[24] R. Hecht-Nielsen, Neurocomputing, (Addison-Wesley, Reading, 1990) pp. 325-330.

[25] H. Martens and T. Naes, Muitivariate Calibration, (John Wiley and Sons, New York, 1989).

[26] J. A. Howell, C. W. Barnes, S. K. Brown, G. W. Flake, R. D. Jones, Y. C. Lee, S. Qian, and R. M. Wright, "Control of a negative-ion accelerator source using neural networks," Nuclear Instruments and Methodr In Physics Research, I A293 517 (1990).

[27] R. D. Jones, "Nonlinear Adaptive Networks: A Little Theory, a Few Applications," । Proceedings of the First Los Alamos Workshop on Cognitive Modeling in System Control: Theoretical Foundations and Prospects for Applications, June 10-14, 1990, Santa Fe, New Mexico. (1990).

[28] M. K. O'Rourke, R. D.Jones, Y. C. Lee, C. W. Barnes, G. W. Flake, K. Lee and S. Qian, "Recurrency in feedforward networks for prediction of high dimensional cliffs," I Los Alamos Report LA-UR-90-2977.

[29] C. W. Barnes, S. K. Brown, G. W. Flake, R. D. Jones,M. K. O'Rourke, and Y. C. Lee, "Applications of neural networks to process control and modeling," Proceedings of the International Conference on Artificial Neural Networks, Helsinki, Finland, June 24-25, 1991, LA-UR-91-126.

[30] W. C. Mead, R. D. Jones, Y. C. Lee, C. W. Barnes, G. W. Flake, L. A. Lee, and M. K. O'Rourke, "Using CNLS-net to predict the Mackey-Glass chaotic time series," LAA-UR-91-324, Proceedings of the International Joint Conference on Neural Networks, July, 1991.

[31] R. D. Jones, C. W. Bames, Y. C. Lee, W. C. Mead, "Information theoretic derivation of network architecture and learning algorithms," LA-UR-91-325, Proceedings of the International Joins Conference on Newral Networks, July, 1991. 
[32] I. Poli and R. D. Jones, "A neural network for prediction of noisy data", LA-UR-91671.

[33] W. C. Mead, R. D. Jones, Y. C. Lee, C. W. Bames, G. W. Flake, L. A. Lee, and M. K. O'Rourke, "Prediction of chaotic time series using CNLS-net: Example: The Mackey-Glass equation," LA-UR-91-720.

[34] J. R. Goulding, “A Neural Network Hybrid Expert System," LA-UR-91-903.

[35] J. R. Goulding, "Adaptive Transfer Functions," LA-UR-91-1004.

[36] C. Barrett, G. Canavan, R. Jones, L. Winter, and M. Wolinsky, "System configuration, tasking, and data fusion in a distributed satellite surveillance system," LA-UR-91.

[37] R. D. Jones, J. R. Goulding, M. A. Norris, and L. Poli, "Foundations of feedforward adaptive computation," LA-UR-91-1478.

[38] R. K. Prasanth, R. D. Jones, and Y. C. Lee, "On the existence and stability of certain types of neuromorphic approximations to functions," LA-UR-91-2881.

[39] W. C. Mead, P. S. Bowling, S. K. Brown, R. D. Jones, C. W. Barnes, H. E. Gibson, J. R. Goulding, and Y. C. Lee, "Optimization and Control of a Small-Angle Negative Ion Source Using an On-Line Adaptive Controller Based on the Connectionist Normalized Local Spline Neural Network," LA-UR-91-3670.

[40] K. R. Bisset and R. L. Kelsey, "Simulation of Traffic Flow and Control Using Conventional, Fuzzy, and Adaptive Methods," Proceedings of the European Simulation Multiconference, York, U.K., June 1-4, 1992, LA-UR-92-1157.

[41] K. R. Bisset and R. L. Kelsey, "Neural Network Adaptation of Fuzzy Membership Functions for Traffic Control".

[42] O. L. Bakalis, R. D. Jones, Y. C. Lee, and B. J. Travis, “A Recurrent Neural Network Approach for the Solution to the Inverse Problem of Iterated Function Systems," LAUR-92-1790.

[43] W. C. Mead, R. D. Jones, Y. C. Lee, C. W. Barnes, G. W. Flake, L. A. Lee, and M. K. O'Rourke "Prediction of chaotic time series using CNLS-Net- Example: The MackeyGlass Equation," in \Nonlinear Modeling and Forecasting, ed. by Martin Casdagli and Stephen Eubank, (Addison Wesley, Redwood City, 1992) p.39.

[44] W. C. Mead, P. S. Bowling, S. K. Brown, R. D. Jones, C. W. Barnes, H. E. Gibson, J. R. Goulding, and Y. C. Lee, "Optimization and control of a small-angle negative ion source using an on-line adaptive controller based on the connectionist normalized local spline network," \Nuclear Instruments and Methods in Physics Research $\backslash$ B72 271, (1992).

[45] Jinqiu Shao, R. D. Jones, and Y. C. Lee, "Orthogonal projection method for fast online learning algorhm of radial basis function neural networks," $\backslash$ Proceedings of the World Conference on Neural Networks III-520, (1993), LA-UR-92-3292. 
[46] R. D. Jones, "Machines the learn: Adaptive computation with neural networks," \Los Alamos Science $\backslash 21$ 195, (1993), This is a special \$50^th\$ anniversary issue.

[47] Suzanne E. Bell, W. C. Mead, R. D. Jones, "Connectionist hyperprism neural network for the analysis of ion mobility spectra: An empirical evaluation," \Journal of Chemical Information and Computer Science $\backslash 33$ 609, (1993).

[48] R. D. Jones, J. Shao, S. V. Coggeshall, K. L. Buescher, "An optimal network architecture for accuracy and generalization," I Scientific Modeling and Computation to be published, LA-UR-93-2474.

[49] J. Shao, R. D. Jones, Robert Tokar, “A robust training algorithm for training CNLS net on erroneous data," LA-UR-93-2475.

[50] W. C. Mead, H. N. Fisher, R. D. Jones, K. R. Bisset, and L. A. Lee, “Application of adaptive and neural network computational techniques to traffic volume and classification monitoring," LA-UR-93-2855.

[51] S. K. Brown, W. C. Mead, P. S. Bowling, R. D. Jones, and C. W. Barnes, “Optimization and control of a small-angle negative ion source using an adaptive neural network controller," $\backslash$ Proceedings of the International Conference on Ion Sources $\backslash 93$ (1993), LA-UR-93-2908.

[52] V. Makhankov, D. Aubrey, D. Cagliostro, R. Jones, B. McVey, and R. Tokar, “A dynamical Model of vehicle motion," LA-UR-93-2914.

[53] R. D. Jones, ed., I Nonlinear Adaptive Computation, monograph, LA-UR-93-3000.

[54] 1. Poli and Roger D. Jones, "Kaiman Filtering in Neural Networks," \Journal of the American Statistical Association, 89117 (1994).

[55] K. L. Buescher and P. R. Kumar, "Selecting Model Complexity in Learning Problems," I 32nd IEEE Conference on Decision and Control, December, 1993.

[56] C. C. Baum, K. L. Buescher, R. D. Jones, K. Lee, M. Messina, D. W. Shevitz, and M. Splichal, "Generalization and Control of a Response surface Model for Plasma Etching", LA-UR-94-205.

[57] R. D. Jones, K. L. Buescher, K. Lee, M. Messina, and M. Splichal, "Generalization of the Response Surface Model for Plasma Etching", LA-UR-93-3813.

[58] R. D. Jones, K. L. Buescher, K. Lee, M. Messina, and M. Splichal, "Sustem Identification and Tracking of a Generalized Plasma Etch Model", LA-UR-93-3865.

[59] R. D. Jones, C. C. Baum, K. L. Buescher, K. Lee, M. J. Messina, D. W. Shevitz, and M. Splichal, "A Simple Control Scheme with Perfect System Identification for Plasma Etching", LA-UR-93-3973.

[60] R. D. Jones, C. C. Baum, K. L. Buescher, K. Lee, M. J. Messina, D. W. Shevitz, and M. Splichal, "Simple Neural Network, Wafer to Wafer Control of Plasma Etching", LAUR-93-4468 
[61] K. L. Buescher, C. C. Baum, R. D. Jones, K. Lee, M. J. Messina, D. W. Shevitz, M. Splichal and R. Caldwell, "Model Predictive Wafer to Wafer Control of Plasma Etching", LA-UR-93-4427.

[62] V. G. Makhankov, R. D. Jones, K. L. Buescher, K. Lee, M. J. Messina, D. W. Shevitz, and M. Splichal, "Physical Featurtes of Plasma in Etching Reactors Reactive Ion Etching (RIE): Part I. The Bulk of the Plasma. Electrons", LA-UR-94-1021.

[63] V. G. Makhankov, R. D. Jones, K. L. Buescher, K. Lee, M. J. Messina, D. W. Shevitz, and M. Splichal, "Physical Featurtes of Plasma in Etching Reactors Reactive Ion Etching (RIE): Part II. The Bulk of the Plasma. Ions, Estimations", LA-UR-94-1652.

[64] K. L. Buescher, R. D. Jones, and M. J. Messina, "Diagnosing Process Faults Using Neural Network Models," LA-UR-93-3598.

[65] R. D. Jones and C. C. Gomez, "Classification Schemes Based on Adaptive Partial Least Squares and Probabalistic Neural Networks," LA-UR-94-1573.

[66] R. D. Jones, S. V. Coggeshall, and C. C. Gomez, "Relationship Between Loss Rate Prediction and K-S Statistic: A Simple Analytic Example," LA-CP-111.

[67] C. C. Baum, P. S. Bowling, S. K. Brown, K. L. Buescher, K. H. Duerre, V. Hanagandi, R. D. Jones, M. J. Messina, W. J. Parkinson, and M. J. Schmitt, "Adaptive Chemical Process Control: Du Pont/ Los Alamos Phase 1 Report," LA-UR-94-1040.

[68] V. Hanagandi, "A Dictionary of Linear Control Schemes: MMC, MPC, DMC, QDMC, and GLC," LA-UR-94-1028.

[69] V. Hanagandi, "Evaluation of Linear Control Strategies for Nonlinear Plants," LA-UR94-1029.

[70] C. C. Baum, K. L. Buescher, R. D. Jones, W. J. Parkinson, and M. J. Schmitt, "Two-Timescale, Model Predictive Control of Two Simulated Chemical Plants: Lagged CSTR and Distillation Column," LA-UR-94-1039.

[71] C. C. Baum and K. L. Buescher, "Description of a Model Predictive Control Software Fackage," LA-UR-719.

[72] W. J. Parkinson and K. H. Duerre, "Neural Networks for Distillation Column Control," LA-UR-902.

[73] V. Hanagandi, "Genetic Algorithms for Controller Design Based on Qualitative Criteria," LA-UR-94-1030.

[74] L. Ke, R. L. Tokar, and B. D. McVey, "An Iniegrated Architecture of Adaptive Neural Network Control for Dynamic Systems," LA-UR-94-1705.

\section{Related Links}

If you are reading this as a hypertext document, you can access related online publications by clicking on related links. 
0.12 Neural Network Announcements and General Information at Los Alamos

0.13 Neural Network Theory

0.14 Related Projects 

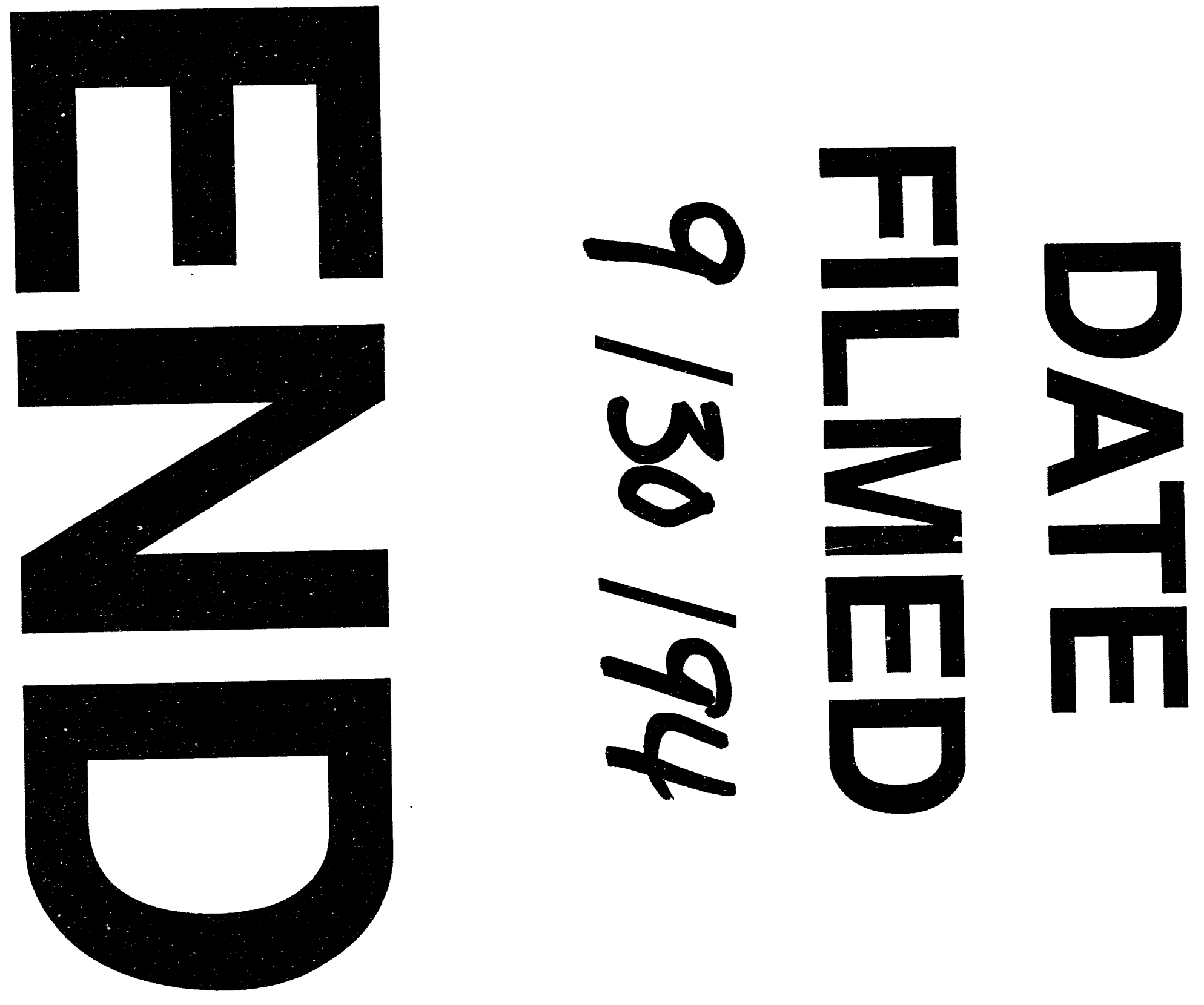
